\section{Avaliação da assistência ao parto em maternidade terciária do interior do Estado de São Paulo, Brasil}

\author{
Evaluation of delivery care in a tertiary \\ maternity in the interior of State of São \\ Paulo, Brazil
}

Fernanda Cristina Manzini 1

Vera Therezinha Medeiros Borges 2

Cristina Maria Garcia de Lima Parada 3

1 Programa de Residência em Saúde da Família. Faculdade de Medicina de Botucatu. Universidade Estadual Paulista "Julio de Mesquita Filho". Botucatu, SP, Brasil.

2 Departamento de Ginecologia e Obstetrícia. Faculdade de Medicina de Botucatu. Universidade Estadual Paulista "Julio de Mesquita Filho". Botucatu, SP, Brasil.

3 Departamento de Enfermagem. Faculdade de Medicina de Botucatu. Universidade Estadual Paulista "Julio de Mesquita Filho". Campus Universitário de Rubião Junior s. n. Botucatu, SP, Brasil. CEP: 18.618-970. E-mail: cparada@fmb.unesp.br

\section{Resumo}

Objetivos: avaliar a estrutura e o processo de assistência ao parto e ao recém-nascido desenvolvidos na maternidade e na unidade de neonatologia de uma instituição hospitalar de nível terciário do interior do Estado de São Paulo, Brasil.

Métodos: estudo descritivo, transversal, voltado para a análise da adesão às normas preconizadas pelo Ministério da Saúde e Organização Mundial da Saúde em relação ao parto, avaliando-se os recursos existentes e as atividades desenvolvidas durante a assistência. Os dados foram colhidos nos anos de 2004 e 2005, a partir de entrevista com o gestor da maternidade, análise de 293 prontuários amostrados e observação de 29 partos realizados.

Resultados: a avaliação da estrutura evidenciou a disponibilidade de equipamentos, instrumentais e medicamentos, de obstetra, pediatra e anestesista e ausência de quartos de pré-parto, parto e puerpério. $\mathrm{Na}$ análise do processo observou-se, entre outras, frequências regulares relacionadas à verificação de pressão arterial e ausculta dos batimentos cardiacos fetais; o preenchimento do partograma foi satisfatório; na assistência ao recém-nascido, foram insatisfatórios o aleitamento na primeira hora de vida e o contato pele a pele.

Conclusões: algumas práticas úteis no parto normal foram pouco utilizadas. Percebe-se uma tendência à incorporação de práticas baseadas em evidências científicas, quando se considerou a realização de procedimentos como tricotomia, enteroclisma e episiotomia, demonstrando uma mudança positiva na assistência ao parto.

Palavras-chave Parto humanizado, Avaliação em saúde, Parto 


\section{Introdução}

A saúde materna e infantil é tema em pauta nos âmbitos nacional e internacional, especialmente no que diz respeito à melhoria da qualidade da atenção. Nesse sentido, o conhecimento do perfil de morbidade e mortalidade de ambos os grupos é essencial para o planejamento das ações na área da saúde. 1,2

As afecções perinatais não só constituem a primeira causa de mortalidade neonatal no país, como também interferem nas mortes ao longo do primeiro ano de vida.1,2 Já a ocorrência de morbidade grave e de morte materna está diretamente relacionada à adequada atenção durante o ciclo gravídico-puerperal, podendo ser significativamente reduzidas se a assistência profissional e a estrutura institucional forem adequadas, independentemente de outras características. $3-5$

Com o objetivo de incrementar a qualidade, ampliar o acesso, a capacidade instalada e a organização da assistência obstétrica e neonatal no Brasil, o Ministério da Saúde instituiu no ano 2000, o Programa de Humanização do Pré-Natal e Nascimento (PHPN), 5,6 que sofreu alguns ajustes em 2005, em virtude da implementação da Política Nacional de Atenção Obstétrica e Neonatal. ${ }^{3}$

Uma das condições básicas para a organização da assistência em saúde é a avaliação dos serviços. No Brasil, desde a Constituição de 1988, cabe aos departamentos ou secretarias municipais de saúde o planejamento sistemático de suas ações, o que requer avaliações periódicas. Assim, essa prática que era quase inexistente há algumas décadas, hoje é uma preocupação das equipes responsáveis pela programação em saúde, possibilitando o desenvolvimento de análises críticas sobre os serviços prestados à população. ${ }^{7}$

Teoricamente pode-se avaliar qualquer intervenção e/ou procedimento, sistema de saúde ou mesmo atividade assistencial, ${ }^{8}$ comparando os resultados obtidos com normas ou outros valores e quantidades que sirvam como parâmetros. Um dos referenciais de avaliação mais utilizados no Brasil foi proposto em 1988 por Donabediam ${ }^{9}$ e compreende a análise da estrutura, processo e resultados.

O componente estrutura avalia os recursos existentes para a execução dos serviços, incluindo os recursos físicos, materiais e humanos, as normas e rotinas estabelecidas, sendo os dados obtidos de registros existentes, por entrevista ou observação local. A avaliação de processo corresponde às atividades realizadas pelos provedores da assistência, referindo-se tanto ao componente técnico quanto ao da relação interpessoal. Por fim, o componente resultado refere-se ao efeito que as ações e procedimentos tiveram sobre a saúde dos usuários ou da comunidade e as relações entre usuários e provedores, intermediadas pelas expectativas de ambos. 9,10

O objetivo desta investigação foi avaliar a estrutura e o processo de assistência ao parto e ao recémnascido, desenvolvidos na maternidade e na unidade de neonatologia de uma instituição hospitalar de nível terciário do interior do Estado de São Paulo.

\section{Métodos}

Trata-se de estudo descritivo e transversal, parte de amplo projeto que avaliou o PHPN nos 31 municípios da Direção Regional de Saúde XI (DIR XI), ${ }^{11}$ sendo voltado para a análise da adesão às normas preconizadas pelo Ministério da Saúde12 e Organização Mundial da Saúde (OMS) ${ }^{13}$ em relação à atenção ao parto e recém-nascido. Esta pesquisa refere-se especificamente ao único serviço terciário da citada regional de saúde, localizado no município de Botucatu, São Paulo, Brasil Tal serviço dispõe de 27 leitos obstétricos, considerando-se as áreas de alojamento conjunto, pré-parto e isolamento. Nele ocorrem, em média, 120 partos mensais. O serviço de neonatologia conta com unidade de cuidados intermediários e unidade de terapia intensiva, totalizando 38 leitos.

A coleta de dados ocorreu nos anos de 2004 e 2005, a partir de três instrumentos construídos com questões fechadas, e foi realizada em duas etapas: na primeira, foi entrevistado o gestor da maternidade, obtendo-se dados sobre sua estrutura e, na segunda, foram observados partos ocorridos, desde a admissão da parturiente até o puerpério, avaliandose a estrutura e o processo de atenção. Concomitante à observação dos partos, realizou-se a análise de prontuários de parturientes e recém-nascidos atendidos na mesma instituição no ano de 2003, pois supunha-se que a presença do observador pudesse provocar mudanças no processo de atenção.

Dois profissionais da área de saúde previamente capacitados observaram os partos ocorridos durante sete dias consecutivos, 24 horas por dia, totalizando 29 partos. Esse procedimento permitiu o acompanhamento de todos os profissionais atuantes na maternidade.

Optou-se pela avaliação dos prontuários do ano de 2003 pela disponibilidade de consultá-los no Setor de Arquivo Médico. A amostra foi estabelecida pela proporção de partos realizados em relação ao 
total de partos ocorridos na DIR XI no mesmo ano (25,5\%), sendo fixado em 293 o número de prontuários de mães e recém-nascidos a serem avaliados (95\% de confiança e $5 \%$ de erro de estimativa). A seleção dos mesmos ocorreu por sorteio dos dias de parto.

Os indicadores de estrutura utilizados foram: para os recursos físicos, a possibilidade de acompanhante e deambulação no pré-parto, a existência de quartos PPP, a adequação das salas de parto normal e cesárea e do alojamento conjunto e berçário para receberem os pais; para os recursos materiais e equipamentos, a existência de material para reanimação neonatal na sala de parto e unidade neonatal, a presença nas salas de parto normal e cesárea de ar comprimido, oxigênio, foco, mesa de parto, carrinho de urgência e de anestesia, estetoscópio de Pinard ou sonar Doppler e berço aquecido e no alojamento conjunto e unidade neonatal, número de berços individuais, roupas suficientes para a mãe e o bebê e a existência de lavabo antes da entrada do berçário. Para os recursos humanos, a presença de médicos (um obstetra e um pediatra para 20 mães e 20 neonatos, respectivamente), enfermeiro (no alojamento conjunto, um a cada 30 binômios e na unidade neonatal a presença deste profissional diariamente em todos os plantões) e auxiliar de enfermagem (um para oito binômios) nos plantões. Para as normas e rotinas, a existência de norma relativa à permanência dos pais no berçário em caso de internação da criança e a existência de protocolo escrito de atenção obstétrica e neonatal.

Os indicadores de processo incluíram: na admissão, a solicitação do cartão ou anotação referente ao pré-natal, informação sobre a possibilidade de acompanhante, aferição da pressão arterial, ausculta dos batimentos cardíacos fetais, aferição de dinâmica uterina, exame de toque vaginal, tricotomia e enteroclisma; no pré-parto, a prescrição de jejum ou repouso, controle não farmacológico da dor, instalação de venóclise, ausculta dos batimentos cardíacos fetais e preenchimento do partograma; na sala de parto, ausculta dos batimentos cardíacos fetais, presença de acompanhante, realização de episiotomia nos partos vaginais, contato pele a pele mãe/bebê e utilização de ocitocina profilática no terceiro estágio do parto e para a assistência ao neonato, atendimento por pediatra na sala de parto, oferecimento de vitamina $\mathrm{K}$, estabelecimento do índice de Apgar no primeiro minuto de vida, idade gestacional por exame físico, identificação do peso, comprimento e perímetro cefálico, coleta de sangue para tipagem sanguínea, anti-HIV e VDRL (Veneral Diseases Research Laboratory) e aleitamento materno na primeira hora de vida; no alojamento conjunto e unidade neonatal, uso de bicos de borracha e leites artificiais rotineiramente e realização de atividades sistemáticas de orientação às mães.

Pelo exposto, os indicadores de estrutura, analisados a partir da entrevista com o gestor da maternidade e da observação realizada pelos profissionais que acompanharam os partos, contemplavam recursos físicos e humanos, materiais e equipamentos e a existência de normas e rotinas. Foram classificados como adequados quando os mesmos estavam de acordo com recomendações do Ministério da Saúde e OMS e inadequados quando insuficientes ou inexistentes.

Para análise do processo da assistência, utilizaram-se dados referentes à observação dos partos e registros dos prontuários, considerando-se duas dimensões: o conjunto de práticas reconhecidamente úteis ao parto normal e que devem ser estimuladas e o conjunto de práticas reconhecidamente prejudiciais ou ineficazes e que devem ser eliminadas.

Consideraram-se, entre as práticas úteis: avaliação do risco gestacional na admissão para o parto, viabilizada pelos dados do cartão pré-natal, oferecimento de líquidos por via oral durante o trabalho de parto e parto, respeito à escolha do acompanhante durante o trabalho de parto, utilização de métodos não farmacológicos para alívio da dor, liberdade de posição e movimento durante o trabalho de parto, utilização do partograma, administração de ocitocina no terceiro estágio do parto e promoção do contato pele a pele mãe/bebê. Considerou-se a proporção de realização do procedimento em relação ao total de partos observados e registros nos prontuários analisados. A qualidade foi satisfatória quando os indicadores mantiveram-se acima de $80 \%$; regular, entre $50 \%$ e $80 \%$ e insatisfatória quando inferior a $50 \% .{ }^{14}$ Porém, especificamente para os indicadores aferição da pressão arterial e da frequência cardíaca fetal e realização de exame sorológico do recém-nascido (VDRL e anti-HIV), por serem procedimentos de baixo custo, mas com alta relação custo/benefício, foram avaliados como satisfatórios apenas quando a cobertura atingiu $100 \%$.

No conjunto de práticas reconhecidamente prejudiciais ou ineficazes, por estarem incluídos indicadores que são desaconselhados, como o uso rotineiro de enteroclisma, tricotomia e infusão endovenosa, classificou-se o desempenho como satisfatório, regular e insatisfatório considerando-se os seguintes parâmetros: abaixo de $10 \%$, de $10 \%$ a $50 \%$ e acima de $50 \%$, respectivamente. 13 
Alguns indicadores de processo foram classificados como adequados ou inadequados. A realização de atividades sistemáticas de orientação às mães foi classificada como adequada e o uso de bicos de borracha e leites artificiais rotineiramente, como inadequado.

Este estudo foi aprovado pelo Comitê de Ética em Pesquisa da Universidade Estadual Paulista "Júlio de Mesquita Filho".

\section{Resultados}

As informações sobre a estrutura para assistência ao parto e neonato constam na Tabela 1.

A análise relativa aos recursos humanos mostrou que os profissionais médicos avaliados estavam disponíveis na maternidade em todos os plantões em proporção adequada ao número de leitos. Com relação à equipe de enfermagem, isso não ocorreu para as enfermeiras, especialmente na área de obstetrícia, mas o número de auxiliares e técnicos de enfermagem estava adequado. Na avaliação dos recursos físicos, destaca-se a inexistência de quartos PPP e a ausência de espaço físico que permitisse a presença de acompanhante ou que a parturiente deambulasse no pré-parto. As observadoras apontaram que duas crianças ocuparam o mesmo berço. Não existiam protocolos escritos para organização da atenção desenvolvida nas áreas de obstetrícia e neonatologia, mas norma escrita autorizava a mãe a permanecer no berçário em caso de internação de seu filho. (Tabela 1)

Quanto ao processo de atenção, os procedimentos realizados na admissão, pré-parto e sala de parto constam da Tabela 2, onde se apresenta separadamente o que foi observado e o que estava registrado nos prontuários amostrados, respectivamente.

Independentemente da adequação ou não de sua realização, excluindo-se o enteroclisma e a episiotomia para parto vaginal, todos os demais

\section{Tabela 1}

\begin{tabular}{|c|c|c|}
\hline Recursos, normas e procedimentos & Sim & Não \\
\hline \multicolumn{3}{|l|}{ Recursos humanos (24 horas/dia) } \\
\hline Proporção adequada de obstetra & $\mathrm{x}$ & \\
\hline Proporção adequada de enfermeira na área obstétrica & & $\mathrm{x}$ \\
\hline Proporção adequada de pediatra & $\mathrm{X}$ & \\
\hline Anestesista & $\mathrm{x}$ & \\
\hline Enfermeira na área neonatal & $\mathrm{x}$ & \\
\hline Proporção adequada de auxiliar de enfermagem & $\mathrm{x}$ & \\
\hline \multicolumn{3}{|l|}{ Recursos físicos } \\
\hline Compatível com a presença de acompanhante no pré-parto & & $\mathrm{x}$ \\
\hline Espaço para deambulação no pré-parto & & $\mathrm{x}$ \\
\hline Existência de quartos pré-parto, parto e puerpério. & & $\mathrm{x}$ \\
\hline Adequação das salas de parto normal e de cesárea & $x$ & \\
\hline Adequação do alojamento conjunto & $\mathrm{x}$ & \\
\hline Adequação do berçário para receber os pais & $\mathrm{x}$ & \\
\hline \multicolumn{3}{|l|}{ Recursos materiais e equipamentos } \\
\hline Equipamentos nas salas de parto normal e de cesárea & $\mathrm{x}$ & \\
\hline Material de reanimação neonatal na sala de parto e berçário & $\mathrm{x}$ & \\
\hline Pinard/sonar doppler na sala de parto & $\mathrm{x}$ & \\
\hline Berço aquecido na sala de parto & $\mathrm{x}$ & \\
\hline Adequação do número de berços do alojamento conjunto & $\mathrm{x}$ & \\
\hline Roupa suficiente para mãe e neonato no alojamento conjunto e berçário & $x$ & \\
\hline Lavabo antes da entrada do berçário & $\mathrm{x}$ & \\
\hline Adequação do número de berços no berçário & & $\mathrm{x}$ \\
\hline \multicolumn{3}{|l|}{ Normas e rotinas } \\
\hline Permissão para a mãe permanecer no berçário & $x$ & \\
\hline Existência de protocolos escritos & & $\mathrm{x}$ \\
\hline
\end{tabular}


Tabela 2

Procedimentos realizados e rotinas adotadas na admissão, pré-parto, parto e com o recém-nascido, segundo os partos observados ( $n=29)$ e os prontuários amostrados (n=293). DIR XI , São Paulo, 2004 a 2005.

\begin{tabular}{|c|c|c|c|c|}
\hline \multirow[t]{2}{*}{ Procedimentos e rotinas } & \multicolumn{2}{|c|}{ Partos observados } & \multicolumn{2}{|c|}{ Prontuários amostrados } \\
\hline & $\mathrm{n}$ & $\%$ & $\mathrm{n}$ & $\%$ \\
\hline \multicolumn{5}{|l|}{ Sala de admissão } \\
\hline Solicitação do cartão ou anotação referente ao pré-natal & 28 & 96,6 & 197 & 67,2 \\
\hline Informação sobre a possibilidade de acompanhante & 16 & 55,2 & 0 & 0,0 \\
\hline Dinâmica uterina & 22 & 75,9 & 153 & 52,2 \\
\hline Toque vaginal & 26 & 89,7 & 231 & 78,8 \\
\hline Pressão arterial & 28 & 96,6 & 253 & 86,3 \\
\hline Ausculta dos batimentos cardíacos fetais & 28 & 96,6 & 255 & 87,0 \\
\hline Tricotomia & 4 & 13,8 & 35 & 11,9 \\
\hline Enteroclisma & 0 & 0,0 & 5 & 1,7 \\
\hline \multicolumn{5}{|l|}{ Sala de pré parto } \\
\hline Indicação de jejum & 18 & 62,1 & 93 & 31,7 \\
\hline Indicação de repouso & 2 & 6,9 & 6 & 2,0 \\
\hline Controle não farmacológico da dor & 4 & 13,8 & 1 & 0,3 \\
\hline Instalação de venóclise & 23 & 79,3 & 199 & 67,9 \\
\hline Ausculta dos batimentos cardíacos fetais & 27 & 93,1 & 263 & 89,8 \\
\hline Preenchimento do partograma & 28 & 96,6 & 278 & 94,9 \\
\hline \multicolumn{5}{|l|}{ Sala de parto } \\
\hline Ausculta dos batimentos cardíacos fetais & 6 & 20,7 & 2 & 0,7 \\
\hline Presença de acompanhante & 11 & 37,9 & 5 & 1,7 \\
\hline Episiotomia para parto vaginal $(n=17 / n=170)$ & 8 & 47,0 & 122 & 71,8 \\
\hline Ocitocina no terceiro estágio do parto & 22 & 75,9 & 172 & 58,7 \\
\hline Contato pele a pele mãe-bebê & 6 & 20,7 & 45 & 15,4 \\
\hline \multicolumn{5}{|l|}{ Assistência ao neonato } \\
\hline Atendimento por pediatra na sala de parto & 28 & 96,6 & 291 & 99,3 \\
\hline Oferecimento de vitamina $\mathrm{K}$ & 29 & 100,0 & 261 & 89,1 \\
\hline Índice de Apgar no primeiro minuto de vida & 29 & 100,0 & 290 & 99,0 \\
\hline Idade gestacional por exame físico & 29 & 100,0 & 288 & 98,3 \\
\hline Identificação do peso & 29 & 100,0 & 291 & 99,3 \\
\hline Identificação do comprimento & 29 & 100,0 & 290 & 99,0 \\
\hline Identificação do perímetro cefálico & 29 & 100,0 & 291 & 99,3 \\
\hline Coleta de sangue para tipagem sangüínea & 28 & 96,6 & 282 & 96,2 \\
\hline Coleta de sangue para exame Veneral Diseases Research Laboratory & 29 & 100,0 & 235 & 80,2 \\
\hline Coleta de sangue para exame anti-HIV & 28 & 96,6 & 74 & 28,7 \\
\hline Aleitamento materno na primeira hora de vida & 6 & 20,7 & 36 & 12,3 \\
\hline
\end{tabular}

procedimentos foram mais frequentemente observados do que anotados. (Tabela 2)

Considerando as práticas que devem ser estimuladas, a observação na admissão evidenciou frequências regulares para a verificação de pressão arterial, ausculta dos batimentos cardíacos fetais e aferição de dinâmica uterina, sendo os demais procedimentos satisfatórios. Porém, pela análise dos prontuários, não se classificou nenhum procedimento como satisfatório quando se consideraram esses indicadores
(Tabela 2).

Entre as práticas danosas, a proporção de tricotomia nos prontuários amostrados e partos observados foi $11,9 \%$ e $13,8 \%$, respectivamente, sendo considerada prática regular. Na sala de pré-parto, o preenchimento do partograma foi satisfatório nas duas situações: análise dos prontuários e observação dos partos e, em contraponto, o controle não farmacológico da dor foi insatisfatório em ambos. Durante a observação dos partos, foram insatisfatórias 
também a indicação de jejum e a instalação de venóclise, sendo este último indicador insatisfatório ainda nos prontuários amostrados. (Tabela 2)

$\mathrm{Na}$ sala de parto, foram insatisfatórios em ambos os casos, a presença de acompanhante, a ausculta dos batimentos cardíacos fetais e o contato pele a pele mãe-bebê, enquanto na assistência ao recémnascido, foi insatisfatório o aleitamento na primeira hora de vida e o índice de coleta de exame de antiHIV. A coleta do VDRL foi insatisfatória apenas nos prontuários amostrados e a realização de episiotomia foi regular nos partos observados e insatisfatória nos prontuários estudados. (Tabela 2)

Observou-se atividade sistemática de orientação às mães e não se presenciou o oferecimento de bicos de borracha e de leites artificiais no alojamento conjunto e unidade neonatal.

\section{Discussão}

Foi possível comparar, para maior parte dos indicadores utilizados, a informação obtida por meio da observação direta da estrutura da maternidade e do processo de atenção ao parto com o registro em prontuários, aumentando a confiabilidade dos resultados, se comparados a outros estudos brasileiros $1,4,6$ com objetivos semelhantes, que, em geral, obtém dados por meio da consulta a prontuários ou entrevista com parturientes e/ou profissionais.

$\mathrm{Na}$ análise das limitações deste estudo deve ser considerado que a avaliação da estrutura utilizou dados fornecidos pelo gestor, que pode ter tido dificuldade de responder de forma imparcial às questões, o que foi relativizado com a inclusão de informações obtidas dos profissionais que observaram os partos.

Destaca-se que a avaliação da estrutura hospitalar não deve ser realizada isoladamente, pois apesar de relacionar-se com a segurança da assistência ao parto, à puérpera e ao neonato, diminuindo a probabilidade de resultados desfavoráveis, não garante a qualidade dos processos. ${ }^{4,15}$

Problemas relativos à área física da instituição estudada, como ausência de espaço para deambulação da parturiente e de quartos PPP, foram identificados como barreiras na implementação de ações preconizadas pela política de humanização da assistência ao parto. A adequação do espaço físico das maternidades pode viabilizar a adoção de práticas úteis para o parto vaginal, como o respeito ao direito da mulher à privacidade no momento do parto e a liberdade de posição e movimentação. 16 Avaliação da qualidade da assistência realizada por mulheres que puderam escolher a posição durante o parto mostrou altos níveis de satisfação, 17 reforçando a necessidade de alterações estruturais para viabilizar tal prática.

A presença do acompanhante junto à parturiente é um direito garantido por lei e estudos evidenciam que este transmite segurança emocional à mulher, trazendo benefícios à sua saúde e do bebê. 5,18 Tais aspectos foram pouco valorizados neste estudo, pois pouco mais da metade das mulheres que tiveram seus partos observados foram informadas sobre a possibilidade de contar com acompanhante durante a internação, embora estudos mostrem a solidão da parturiente na ausência deste. 18,19

Não foi avaliada a área física disponível para cada binômio, mas pode-se afirmar que o número de berços era inadequado, visto que dois bebês ocupavam o mesmo berço. Essa prática pode interferir na segurança e bem-estar do neonato por facilitar a ocorrência de infecções cruzadas, com consequente aumento na taxa de mortalidade infantil.20

Ainda com relação à estrutura, no que se refere aos equipamentos, instrumentais e medicamentos, foram considerados adequados, tanto no alojamento conjunto quanto nas salas de parto. Essa situação é privilegiada quando comparada à relatada em estudos realizados em outros países subdesenvolvidos, onde a falta de equipamentos e medicamentos mostrou-se rotineira. 17,19

A avaliação foi satisfatória em relação a disponibilidade de material para reanimação neonatal, tanto na sala de parto quanto no berçário. Essa situação difere da encontrada em outros Estados brasileiros, pois estudos apontam a indisponibilidade desses materiais. 4,21

Todo serviço de assistência ao parto tem que dispor de profissionais habilitados para o desenvolvimento das práticas obstétricas, visando garantir um parto seguro. ${ }^{5}$ A maternidade avaliada contava com a presença de docentes e médicos residentes nas áreas de obstetrícia, pediatria e anestesiologia ininterruptamente nas 24 horas do dia, permitindo afirmar que do ponto de vista médico os recursos necessários a um serviço terciário estavam adequados. ${ }^{22}$ Considerando a situação das maternidades brasileiras, em 2002 um terço delas não tinha obstetra nem mesmo à distância e $74 \%$ não contavam com a presença de pediatra. ${ }^{23}$ No Estado do Maranhão evidenciou-se que aproximadamente $40 \%$ dos partos haviam sido assistidos por auxiliares de enfermagem e parteiras. ${ }^{14}$

Não estava disponível um enfermeiro para 30/binômio, em todos os plantões na área obstétrica, considerando-se centro obstétrico e alojamento 
conjunto. Na área neonatal, apenas avaliou-se a presença desse profissional nas 24 horas do dia sem, entretanto, considerar a proporção de leitos ou mesmo taxa de ocupação desses. Assim, a situação privilegiada relacionada aos médicos não se repetiu com o enfermeiro. Estudo realizado na regional de saúde onde se insere o serviço que se está avaliando, com objetivo de analisar a assistência pré-natal e ao parto, evidenciou atendimento centrado no trabalho médico. ${ }^{24}$

A ação do enfermeiro na assistência ao parto pode possibilitar a construção de um relacionamento terapêutico, pautado no estabelecimento de vínculo e confiança, que em conjunto com as ações médicas, permitem a condução de um trabalho de parto resolutivo e menos intervencionista. 25 É recomendado que o obstetra esteja mais voltado para a atenção ao parto de alto risco e que os de baixo risco sejam assistidos por enfermeiros especialistas. 14 Assim, mesmo considerando-se que o serviço estudado é de nível terciário, a ausência de enfermeiros no processo de atenção de forma efetiva expõe certa fragilidade do serviço.

Protocolos de assistência representam conhecimento científico acumulado, facilitando o funcionamento do serviço 10 e auxiliando na organização do processo de trabalho. A instituição estudada, apesar de tratar-se de hospital de ensino, não dispunha deste importante instrumento de gestão, o que pode levar ao desconhecimento dos fluxos e condutas por parte dos profissionais envolvidos na assistência.

Quanto à avaliação dos indicadores de processo, apresentaram maiores frequências na observação dos partos do que nos registros dos prontuários. Considera-se que a presença do observador pode ter otimizado a forma como os cuidados foram prestados.

Na observação, desde a admissão até o parto, evidenciou-se que entre as práticas úteis ao parto normal, foram satisfatórios os indicadores realização do exame de toque vaginal e solicitação de cartão de pré-natal, melhor situação que a apontada por estudo realizado na África do Sul, em que informações sobre o pré-natal foram solicitadas para apenas $15 \%$ das gestantes. 26

Conhecer a evolução pré-natal contribui com o planejamento da assistência ao parto a ser desenvolvida, facilitando o processo de atenção. Além disso, tratando-se de hospital de ensino, há certa rotatividade de alunos e médicos residentes, de forma que a assistência ao parto não necessariamente é realizada pela pessoa que fez a admissão, o que ratifica a importância da qualidade dos registros nos prontuários.
Embora tenham sido considerados regulares nos partos observados os indicadores aferição da pressão arterial e ausculta dos batimentos cardíacos fetais na admissão, deve-se ponderar que sua ausência esteve relacionada à internação no período expulsivo. A proporção obtida, 96,6\% em ambos os casos, foi superior a de maternidades privadas indianas, onde a proporção foi de $77 \%$ e $70 \%$, respectivamente. 19

No pré-parto, a prática universal de ausculta intermitente dos batimentos cardíacos fetais foi classificada como regular, por não ter atingido cobertura de $100 \%$. A situação, porém, foi melhor que a observada em maternidades de Belo Horizonte, onde em quase metade dos prontuários esse indicador estava ausente. ${ }^{1}$ A falta de registro de um determinado procedimento pode indicar falha importante no processo de assistência, uma vez que quando não anotados, não podemos garantir que foram realizados. 7

O controle não farmacológico da dor foi considerado insatisfatório, sugerindo que tal prática não foi incorporada no processo da assistência. A sensação de dor referida deve ser respeitada, pois para muitas mulheres o parto é sinônimo de dor e sofrimento, envolvendo a necessidade de ajuda e apoio. Embora não existam evidências científicas que apóiem a indicação de técnicas não farmacológicas, há relatos positivos de mulheres que as consideraram úteis e reconfortantes. 13,27

A utilização do partograma é obrigatória em todas as maternidades conveniadas com o Sistema Único de Saúde (SUS)12 e está incorporada à rotina do serviço avaliado. Essa situação também difere da encontrada em maternidades do Estado do Maranhão: em apenas $13,3 \%$ dos partos observados esse instrumento estava preenchido. 14

Com relação aos procedimentos que devem ser evitados no parto, teve desempenho satisfatório a realização de enteroclisma, regular a realização de tricotomia e insatisfatória a taxa de instalação de venóclise, sendo esta situação melhor que a de estudo realizado na Índia, onde se encontraram índices elevados dessas intervenções tanto em maternidades públicas quanto privadas, embora piores nestas últimas. ${ }^{19}$

A episiotomia para partos vaginais foi regular e insatisfatória, quando se consideraram os partos observados e os prontuários amostrados, respectivamente. As frequências desse procedimento encontradas nesse estudo, mesmo estando acima dos índices recomendados pela publicação da OMS, 13 apresentaram-se inferiores quando comparadas a uma maternidade escola da cidade de São Paulo. 28

Em relação às práticas comprovadamente úteis, 
na observação dos partos e nos prontuários analisados, foram considerados insatisfatórios os quesitos presença de acompanhante e contato pele a pele mãe/bebê. Estudo realizado em maternidade pública para avaliar a participação dos pais no parto mostrou que a maioria havia sido informada dessa possibilidade apenas no momento da internação da gestante, não podendo participar por não estarem preparados. 18

No que diz respeito ao contato cutâneo direto e precoce entre mãe e filho, embora seja prática comprovadamente benéfica, simples e sem custos, foi pouco utilizada, o mesmo ocorrendo em maternidade do sul do Brasil,29 sinalizando que sua adoção rotineira requer mudança de postura profissional, com valorização da humanização do cuidado.

Atividades educativas são realizadas rotineiramente na maternidade, podendo constituir espaço para troca de saberes e vivências relativas à parturição e viabilizando a construção de vínculo entre profissionais e puérperas. A não utilização de bicos de borracha e leite artificial no alojamento conjunto e unidade neonatal evidencia o empenho institucional na promoção do aleitamento materno. 30

Investigação realizada na África do Sul evidenciou que $71 \%$ das mães foram encorajadas a amamentar precocemente, 26 situação melhor que a da maternidade estudada, já que nesta o aleitamento na primeira hora de vida foi insatisfatório tanto nos prontuários quanto nos partos observados. No mesmo serviço, em 2001, profissionais que atuavam no centro obstétrico apontaram a falta de preparo técnico para essa ação. ${ }^{31}$

Em síntese, algumas práticas demonstradamente úteis no parto ainda são pouco implementadas na maternidade estudada, enquanto outras prejudiciais ou ineficazes continuam sendo realizadas, talvez por tratar-se de serviço terciário. Porém, percebe-se tendência à humanização e incorporação de evidências científicas na assistência.

Oferecer assistência humanizada conforme proposto pelo PHPN parece ser o grande desafio que se apresenta aos profissionais dessa maternidade. É necessária uma nova abordagem, que considere e estimule a participação ativa da mulher e de seu acompanhante, que viabilize a atuação do enfermeiro na equipe e o uso de técnicas não farmacológicas para alívio da dor.

Os resultados deste estudo podem contribuir para reflexão e debate entre os profissionais envolvidos no cuidado e gestores, apontando fragilidades e fortalezas e indicando caminhos a serem seguidos com o objetivo de melhorar a assistência ao parto e ao recém-nascido.

\section{Referências}

1. Lansky S, França E, César CC, Neto LCM, Leal MC. Mortes perinatais e avaliação da assistência ao parto em maternidades do Sistema Único de Saúde em Belo Horizonte, Minas Gerais, Brasil, 1999. Cad Saúde Pública. 2006; 22: 117-30.

2. Monteiro RA, Schmitz BAS. Principais causas básicas da mortalidade infantil no Distrito Federal, Brasil: 1999 a 2000. Rev Bras Saúde Matern Infant. 2004; 4: 413-21.

3. Brasil. Ministério da Saúde. Instituição da Política Nacional de Atenção Obstétrica e Neonatal. Portaria n.o1067 de 4 de julho de 2005. Diário Oficial da República Federativa do Brasil (DOU). Brasília, DF. 6 jul. 2005, Seção I, p.25-30.

4. Costa JO, Xavier CC, Proietti FA, Delgado MS. Avaliação dos recursos hospitalares para assistência perinatal em Belo Horizonte, Minas Gerais. Rev Saúde Pública. 2004; 38 : 701-8.

5. Brasil. Ministério da Saúde. Institui o Programa de Humanização do Pré-natal e Nascimento. Portaria GM/569, de 01 de junho de 2000. Diário Oficial da República Federativa do Brasil (DOU). Brasília, DF. 18 agosto 2000, Seção 1, p.112.

6. Deslandes SF. A ótica de gestores sobre a humanização da assistência nas maternidades municipais do Rio de Janeiro. Cienc Saúde Coletiva. 2005; 10: 615-26.
7. Scochi MJ. Indicadores da qualidade dos registros e da assistência ambulatorial em Maringá, (Estado do Paraná, Brasil), 1991: um exercício de avaliação. Cad Saúde Pública. 1994; 10: 356-67.

8. Pereira MG. Qualidade dos serviços de saúde. In: Pereira MG. Epidemiologia: teoria e prática. 5 ed. Rio de Janeiro: Guanabara Koogan; 2001. p. 538-60.

9. Donabedian A. The quality of care - how come it be assessed? J Am Med Assoc. 1988; 260: 1743-8.

10. Rattner D. A epidemiologia na avaliação da qualidade: uma proposta. Cad Saúde Pública. 1996; 12 (Supl 2): 21-32.

11. Parada CMGL. Avaliação do Programa de Humanização do Pré-Natal e Nascimento (PHPN) na DIR XI - Botucatu. [livre-docência]. Ribeirão Preto, São Paulo: Escola de Enfermagem de Ribeirão Preto da Universidade de São Paulo; 2006.

12. Brasil. Ministério da Saúde. Secretaria de Políticas de Saúde. Parto, aborto e puerpério: assistência humanizada à mulher. Brasília, DF; 2003.

13. OMS (Organização Mundial da Saúde). Maternidade Segura. Assistência ao parto normal: um guia prático. Genebra; 1996. (Publicação OMS/SRF/MSM/96.24). 
14. Alves MTSSB, Silva AAM. Avaliação de qualidade de maternidades: assistência à mulher e ao seu recém-nascido no Sistema Único de Saúde. São Luis: UNICEF; 2000.

15. Marchisio S, Ferraccioli K, Barbieri A, Porcelli A, Massimiliano P. Care pathways in obstetrics: the effectiveness in reducing the incidence of episiotomy in childbirth. J Nurs Manag. 2006; 14: 538-43.

16. Domingues RMSM, Santos EM, Leal MC. Aspectos da satisfação das mulheres com a assistência ao parto: contribuição para o debate. Cad Saúde Pública. 2004; 20 (Supl 1): 52-62.

17. Peabody JW, Gertler PJ, Leibowitz A. The policy implications of better structure and process on birth outcomes in Jamaica. Health Policy. 1998; 1-13.

18. Carvalho MLM. Participação dos pais no nascimento em maternidade pública: dificuldades institucionais e motivações dos casais. Cad Saúde Pública. 2003; 19 (Supl 2): S389-98.

19. Hulton LA, Mattheus Z, Stones RW. Applying a framework for assessing the quality of maternal health services in urban India. Social Sci Med. 2007; 64: 2083-95.

20. Oliveira MIC, Leal MC. Alojamento conjunto e parto cesáreo no Estado do Rio de Janeiro. Rev Saúde Pública. 1997; 31: 572-80

21. APM (Associação Paulista de Medicina), CREMESP (Conselho Regional de Medicina do Estado de São Paulo). $\mathrm{CQH}$ : Programa de controle de qualidade do atendimento médico hospitalar do Estado de São Paulo: manual de orientação aos hospitais. São Paulo; 1998.

22. Brasil. Ministério da Saúde. Departamento Nacional de Auditoria do SUS. Coordenação de Sistemas de Informação SUS - Legislação Federal. Portaria n ${ }^{\circ} 1016$, de 26 de Agosto de 1993. Alojamento conjunto. Diário Oficial da União (DOU). Brasília, DF, 1 set. 1993, Seção I, p.13066.

Recebido em 21 de janeiro de 2008

Versão final apresentada em 7 de janeiro de 2009

Aprovado em 20 de janeiro de 2009
23. Leal MC, Viacava F. Maternidades do Brasil. Radis: Com Saúde. 2002; (2): 8-26.

24. Parada CMGL. Avaliação da assistência pré-natal e puerperal em região do interior do Estado de São Paulo em 2005. Rev Bras Saúde Matern Infant. 2008; 8: 113-24.

25. Dias MAB, Domingues RMSM. Desafios na implantação de uma política de humanização da assistência hospitalar ao parto. Cienc Saúde Coletiva. 2005; 10: 669-705.

26. Mngadi PT, Thembi ITT, Ahlberg BM. Quality of maternity care for adolescent mothers in Mbabane, Swaziland. Int Nurs Rev. 2000; 49: 38-46.

27. Wright ME, McCrea $H$, Stringer M, Murphy-Black $T$. Personal control in pain relief during labour. J Adv Nurs. 2000; 32: 1168-77.

28. Oliveira SMJV, Miquilini EC. Frequência e critérios para indicar a episiotomia. Rev Esc Enferm USP. 2005; 39: 28895.

29. Reis AE, Patrício ZM. Aplicação das ações preconizadas pelo Ministério da Saúde para o parto humanizado em um hospital de Santa Catarina. Cienc Saúde Coletiva. 2005;10 (Supl): 221-30.

30. Lamounier JA. O efeito de bicos e chupetas no aleitamento materno. J Pediatr. (Rio J). 2003; 79: 284-6.

31. Manzini Fernanda C, Parada Cristina MGL, Juliani Carmen MCM. Aleitamento materno na sala de parto: a visão dos profissionais de saúde. In: Anais do VIII Simpósio Brasileiro de Comunicação em Enfermagem [anais online]; 2002 maio 2-3; São Paulo, SP, Brasil. 2002 [consultado em: 19 ago 2004]. Disponível em: URL: http://www.proceedings.scielo. br/scielo. php?script=sci_arttext\&pid=MSC00000000520020 00200024\&lng=pt\&nrm $=$ van. 